\title{
Effect of Intravitreal Anti-Vascular Growth Factor Agents With or Without Macular Photocoagulation on Diabetic Macular Edema: A Systematic Review and Meta-Analysis
}

\author{
Lanjun Cui · Bingtian Jiao • Quanhong Han
}

Received: February 22, 2019 / Published online: May 11, 2019

(C) The Author(s) 2019

\begin{abstract}
Introduction: Diabetes is a common cause of new sight loss in populations world-wide, and diabetic macular edema (DME) is a major cause of visual deficits in the diabetic populations of developed countries. We have performed a meta-analysis to evaluate whether combined treatment with anti-vascular endothelial growth factor (VEGF) injections and macular photocoagulation (MPC) is more efficacious than primary monotherapy with anti-VEGF injections in patients with DME.

Methods: We systematically searched the PubMed and Web of Science databases for studies providing sufficient information for a comparison of pre- and post-treatment of central macular thickness (CMT) and best-corrected
\end{abstract}

Enhanced Digital Features To view enhanced digital features for this article go to https://doi.org/10.6084/ m9.figshare.8053310.

Electronic Supplementary Material The online version of this article (https://doi.org/10.1007/s13300019-0631-5) contains supplementary material, which is available to authorized users.

L. Cui · B. Jiao (₫)

Department of Ophthalmology, Shandong

Provincial Dezhou Youfu Hospital, Dezhou 253000,

Shandong, China

e-mail: Jiaobingtian@163.com

Q. Han

Tianjin Eye Hospital, Tianjin 300041, China visual acuity (BCVA) between two groups of patients with DME given interventional therapies (monotherapy with an anti-VEGF agent vs. combination therapy with an anti-VEGF agent and MPC) before January 2019. A meta-analysis was performed to summarize the results of the studies included in the systematic review.

Results: The results of our meta-analysis indicated that post-treatment CMT was significantly lower at 3 months in DME patients receiving combination therapy with bevacizumab, a humanized anti-VEGF antibody, and MPC than in those receiving monotherapy with bevacizumab. The results also showed that posttreatment CMT was lower in DME patients given ranibizumab, an anti-VEGF agent, in combination with MPC at 6,9 and 12 months than in those treated with ranibizumab alone. However, no significant differences were found in post-treatment BCVA at 1, 3, 6, 9 and 12 months between DME patients receiving combination therapy with an anti-VEGF agent (bevacizumab or ranibizumab) and MPC and those receiving monotherapy with an antiVEGF agent.

Conclusion: In conclusion, the results of our meta-analysis demonstrate a transiently synergistic effect of MPC on CMT when this treatment is combined with anti-VEGF agents, whereas no similar synergistic effect could be detected on the BCVA. A relatively longer follow-up was essential to be able to evaluate the long-term existence of this synergistic effect. 
Keywords: Anti-vascular growth factor agents; Diabetic macular edema; Macular photocoagulation

\section{INTRODUCTION}

Diabetic macular edema (DME) is a condition that results from fluid accumulation in the macula and cystoid edema of the macula, usually accompanied by exudate deposition. It can be attributed to diabetic retinopathy, which causes swelling in the macula [1]. With the steady increase in the prevalence of diabetes world-wide, DME is considered to be one of the main causes of visual deficits in the populations of developed countries, affecting $7 \%$ of diabetic patients [2, 3].

The traditional treatments of DME are intravitreal triamcinolone, macular photocoagulation (MPC) and pars plana vitrectomy, in combination with adequate control of the diabetes and hypertension [4]. More recently, antivascular endothelial growth factor (anti-VEGF) injections have become the standard of care for eyes with DME and vision impairment [5]. In the past decade, a number of studies have shown that anti-VEGF injections are effective in reducing central macular thickness (CMT) and improving best-corrected visual acuity (BCVA) $[6,7]$. However, anti-VEGF agents are not able to treat macular hypoxia, which is the main cause of DME [6], and thus the effects of antiVEGF agents are transient. In addition, the duration of action of anti-VEGF agents is limited, as exemplified by the short half-life of bevacizumab and ranibizumab, both anti-VEGF agents, in eyes of 9.8 days and 2.75 days, respectively, resulting in the recurrence of macular edema in eyes treated with anti-VEGF agents within a few weeks [8-11]. Laser photocoagulation has been shown to cause damage to some, but not all photoreceptors and to reduce the high oxygen consumption of the photoreceptors by a pathway different from that of antiVEGF agents [12]. Consequently, the combination of MPC and anti-VEGF injections may be an effective therapy to prolong the effectiveness of anti-VEGF agents and contribute to a better result by reducing macular hypoxia. In addition, the effectiveness of MPC may be enhanced by MPC becoming easier due to to a reduction of macular edema by anti-VEGF injections. A number of recent studies have indicated that treatment with anti-VEGF agents in combination with MPC is superior to therapy with an anti-VEGF agent alone in terms of reducing CMT and improving BCVA in patients with DME [9, 13]. However, other studies have reported that there was no significant difference in CMT reduction and BCVA improvement between DME patients given anti-VEGF agents in combination with MPC and those given antiVEGF agents alone [14, 15].

We have therefore performed a meta-analysis to evaluate whether combination therapy with anti-VEGF agents and MPC is more efficacious than primary monotherapy with anti-VEGF injections in terms of DME treatment.

\section{METHODS}

The present study was conducted according to the Preferred Reporting Items for Systematic Reviews and Meta-Analyses (PRISMA) statement [16].

\section{Compliance with Ethics Guidelines}

This article is based on previously conducted studies and does not contain any studies with human participants or animals performed by any of the authors.

\section{Search Strategy, Inclusion Criteria, Exclusion Criteria and Data Collection}

We performed a systematic search of the PubMed and Web of Science databases for articles written in English and published up to January 2019. The search terms used were: "bevacizumab" OR "avastin" OR "aflibercept" OR "ranibizumab" OR "conbercept" OR "antiVEGF" OR "anti-vascular endothelial growth factor" AND "photocoagulation" AND "diabetic 
macular edema". This search strategy resulted in the identification of 354 articles once duplicate studies had been excluded.

The inclusion criteria were randomized controlled trials (RCTs) or high-quality comparative studies that provided sufficient data for a comparison of pre- and post-treatment CMT and BCVA between two groups of DME patients given interventional therapies (anti-VEGF agents alone vs. combination therapy of antiVEGF agents and with MPC).

The exclusion criteria were: (1) articles which did not provide sufficient data on pre- and posttreatment CMT and BCVA; (2) reviews, metaanalyses and case studies.

Two reviewers independently extracted the following data: author, publication year, research type, location of the study, number of eyes and mean age of patient, information on treatment (dosage of anti-VEGF agents, mean time of anti-VEGF agents during follow-up, and type of MPC), duration of follow-up and treatment outcome.

\section{Meta-Analysis}

The weighted mean difference in CMT and BCVA with the 95\% confidence interval was obtained using STATA version 12.0 software (StataCorp LP, College Station, TX, USA) to estimate the treatment effect. The $Q$ test and $I 2$ were applied to evaluate the heterogeneity between included studies. Random effects models were used when there was high heterogeneity ( $p$ value for $Q$ test $\leq 0.05$ ), otherwise, fixed effects models were used. A sensitivity analysis was performed by removing one individual study each time to evaluate the stability of the meta-analysis. Additionally, subgroup analyses (for different anti-VEGF agents and different study designs) were conducted to explore the impact of heterogeneous types of anti-VEGF agents and research types on the heterogeneity of the meta-analysis. Sensitivity analyses were also conducted to evaluate the stability of the meta-analysis. Publication bias was evaluated using Begg's test, Egger's test and funnel plots.

\section{RESULTS}

\section{Search Results}

A flow chart showing the results of the initial research and subsequent progression in the selection process is given in Fig. 1 . Table 1 shows the characteristics and results of the 12 studies ultimately included in the meta-analysis. All 12 studies (10 RCTs, 2 retrospective studies) compared post-treatment CMT and BCVA between two groups of DME patients given interventional therapies (monotherapy with an anti-VEGF agent vs. combination therapy with an anti-VEGF agent and MPC) and included 748 patients who received monotherapy with an anti-VEGF agent and 663 patients who were given combination therapy with an anti-VEGF agent and MPC. Of the 12 studies, five used bevacizumab as the anti-VEGF agent, six used ranibizumab, and one used conbercept.

\section{Meta-Analysis Results}

The meta-analysis indicated that post-treatment CMT at 3, 6 and 9 months was significantly lower in DME patients given combination therapy with an anti-VEGF agent and MPC than in DME patients receiving monotherapy with an anti-VEGF agent, but that no significant differences in CMT were detected between two groups at 1 and 12 months of follow-up (see Fig. 2). Subgroup analyses showed that DME patients given bevacizumab in combination with MPC showed significantly lower posttreatment CMT at 3 months than did those given bevacizumab alone, whereas no significant differences in post-treatment CMT were detected between the two groups at 6 and 12 months. However, DME patients given combination therapy with ranibizumab and MPC showed significantly lower post-treatment CMT at 6, 9 and 12 months than did those receiving monotherapy with ranibizumab, whereas no significant differences in posttreatment CMT were detected between the two groups at 3 months (see Fig. 3). Subgroup analyses showed that the results were the same when only the ten RCTs were included in the 

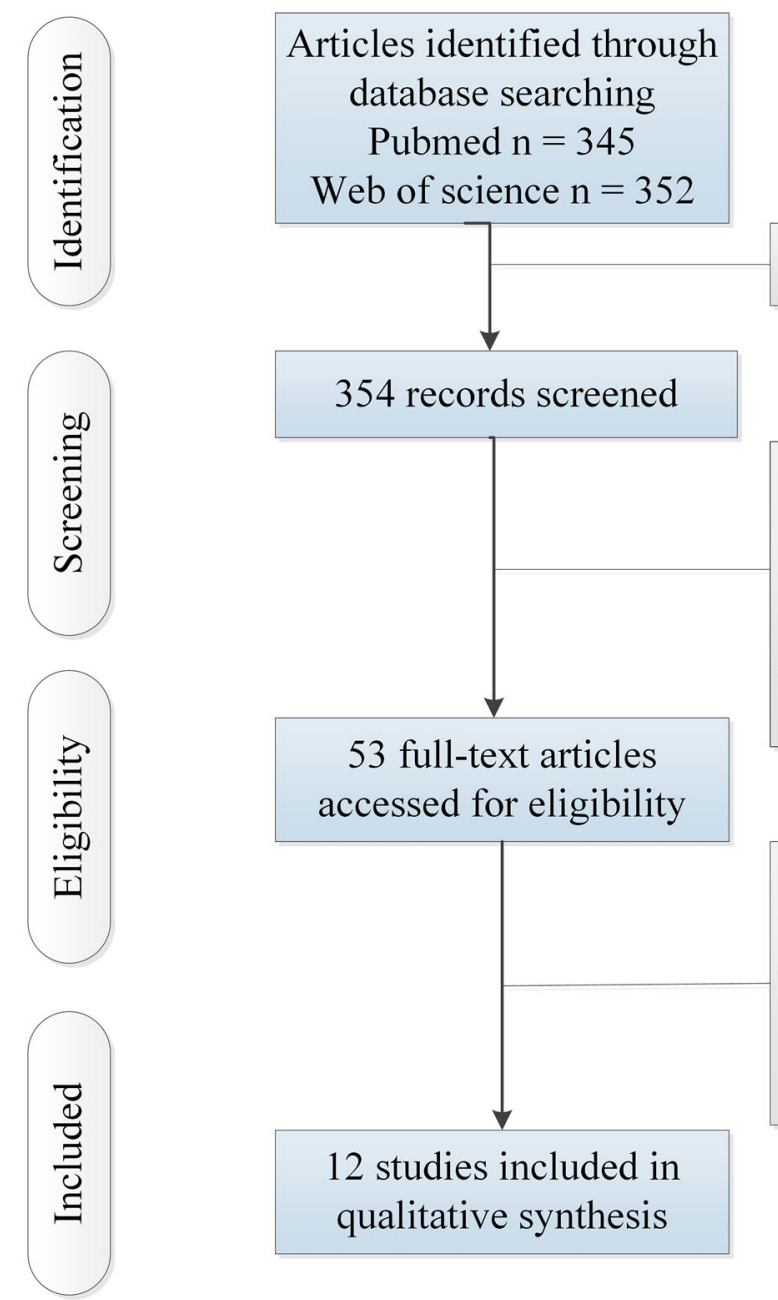

343 duplicates removed

Studies not associated with DME and anti-VEGF agents were excluded; Animal studies were excluded; Reviews, case studies, guidelines, meeting abstracts were excluded. $(\mathrm{n}=301)$

Fig. 1 Flow chart of the systematic review process for the selection of articles to be included in the meta-analysis. $D M E$ Diabetic macular edema, $M P C$ macular photocoagulation, $V E G F$ vascular endothelial growth factor

meta-analysis and when all 12 studies were included (see Fig. 4).

Our meta-analysis showed no significant differences in post-treatment BCVA at 1, 3, 6, 9 and 12 months between DME patients given combination therapy with an anti-VEGF agent and MPC and those given monotherapy with an anti-VEGF agent (see Fig. 5). The subgroup analyses did not detect any significant differences in post-treatment BCVA between the two groups in terms of anti-VEGF agent administered (bevacizumab or ranibizumab; see Fig. 6). In addition, the meta-analysis including only the RCTs revealed no significant differences in post-treatment BCVA between the two patients groups at all follow-up points (see Fig. 7).
The sensitivity analysis showed no changes in the direction of effect when any one study was excluded for all meta-analyses at different follow-up points (see Electronic Supplementary Material [ESM] Fig. 1). Moreover, Begg's test, Egger's tests and funnel plots did not detect any significant risk of publication bias for all metaanalyses at different follow-up points (see ESM Table 1 and Fig. 2).

\section{DISCUSSION}

The results of our meta-analysis indicated that post-treatment CMT was significantly lower at 3 months of follow-up in DME patients given 


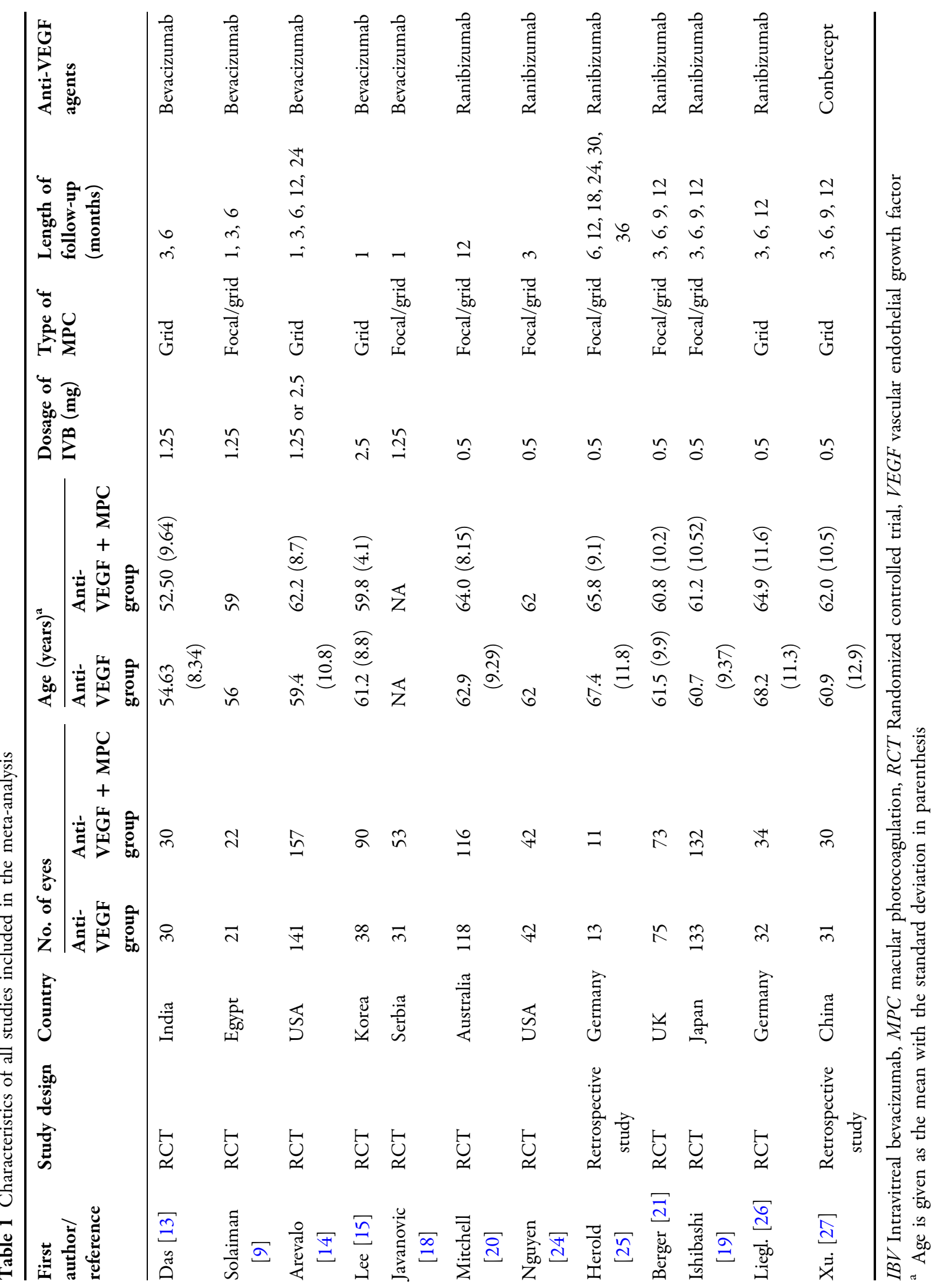


Study ID

WMD $(95 \% \mathrm{Cl})$

1 month

Solaiman et al. (2010)

Arevalo et al. (2013)

Lee et al. (2011)

Jovanovic et al. (2015)

Subtotal (I-squared $=69.7 \%, p=0.019$ )

3 months

K. Das et al. (2018)

Solaiman et al. (2010)

Arevalo et al. (2013)

Berger et al. (2015)

Ishibashi et al. (2015)

Liegl et al. (2014)

Xu et al. (2016)

Subtotal (l-squared $=88.2 \%, p=0.000)$

6 months

K. Das et al. (2018)

Solaiman et al. (2010)

Arevalo et al. (2013)

Herold et al. (2018)

Berger et al. (2015)

Ishibashi et al. (2015)

Liegl et al. (2014)

Xu et al. (2016)

Subtotal (I-squared $=87.6 \%, p=0.000)$

9 months

Berger et al. (2015

Ishibashi et al. (2015)

Xu et al. (2016)

Subtotal (l-squared $=0.0 \%, p=0.814$ )

12 months

Arevalo et al. (2013)

Mitchell et al. (2011)

Herold et al. (2018)

Berger et al. (2015)

Ishibashi et al. (2015)

Liegl et al. (2014)

Xu et al. (2016)

Subtotal (I-squared $=84.9 \%, p=0.000)$

18 months

Herold et al. (2018)

Subtotal $(\mathrm{l}$-squared $=. \%, p=$.)

24 months

Arevalo et al. (2013)

Herold et al. (2018)

Subtotal (I-squared $=88.5 \%, p=0.003$ )

30 months

Herold et al. (2018)

Subtotal $(\mathrm{l}$-squared $=. \%, p=$.)

36 months

Herold et al. (2018)

Subtotal $(\mid$-squared $=. \%, p=$.)

Overall $(\mathrm{I}-$ squared $=85.6 \%, p=0.000)$

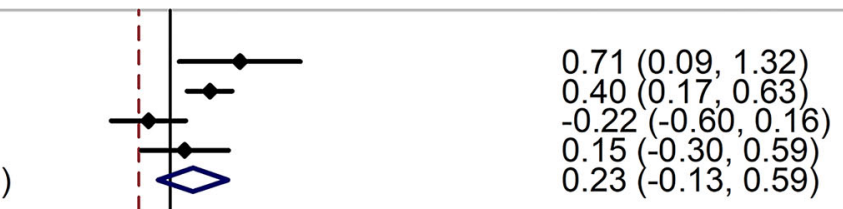

$-1.03(-1.57,-0.49)$

$-2.88(-3.75,-2.02)$

$-0.21(-0.44,0.02)$

$-0.19(-0.51,0.13$

$-0.09(-0.33,0.15)$

$-0.72(-1.22,-0.22)$

$0.14(-0.36,0.64)$

$-0.59(-1.00,-0.17)$

$-1.43(-2.00,-0.86)$

$-2.51(-3.31,-1.70)$

$0.07(-0.16,0.29)$

$-0.43(-1.24,0.39)$

$-0.11(-0.43,0.21$

$-0.27(-0.51,-0.03)$

$-0.12(-0.60,0.37)$

$-0.53(-1.04,-0.02)$

$-0.58(-0.98,-0.17)$

$-0.25(-0.57,0.07)$

$-0.30(-0.54,-0.05)$

$-0.45(-0.95,0.06)$

$-0.30(-0.48,-0.12)$

$0.57(0.34,0.80)$

$-0.17(-0.43,0.08)$

$-0.60(-1.42,0.23)$

$-0.38(-0.71,-0.06)$

$-0.30(-0.54,-0.05)$

$-0.33(-0.82,0.16)$

$-0.16(-0.67,0.34$

-0.16 (-0.49, 0.18)

$-0.96(-1.82,-0.11)$

$-0.96(-1.82,-0.11)$

$0.52(0.29,0.75)$

$-0.79(-1.62,0.05)$

$-0.07(-1.34,1.20)$

$-0.31(-1.12,0.50)$

$-0.31(-1.12,0.50)$

$-0.69(-1.51,0.14)$

$-0.69(-1.51,0.14)$

$-0.32(-0.49,-0.15)$ 
4 Fig. 2 Forest plot of central macular thickness (CMT) comparing the two patient groups (DME patients given combination therapy with an anti-VEGF agent and MPC vs. those receiving monotherapy with an anti-VEGF agent) at $1,3,6,9,12,18,24,30,32$ and 36 months of follow-up. Diamonds denote weighted mean difference $(W M D)$ and 95\% confidence interval $(C I)$. Negative values indicate lower CMT in DME patients given combination therapy with an anti-VEGF agent and MPC than in DME patients receiving monotherapy with an anti-VEGF agent.

combination therapy with bevacizumab and MPC than in DME patients receiving monotherapy with bevacizumab. In addition,

A

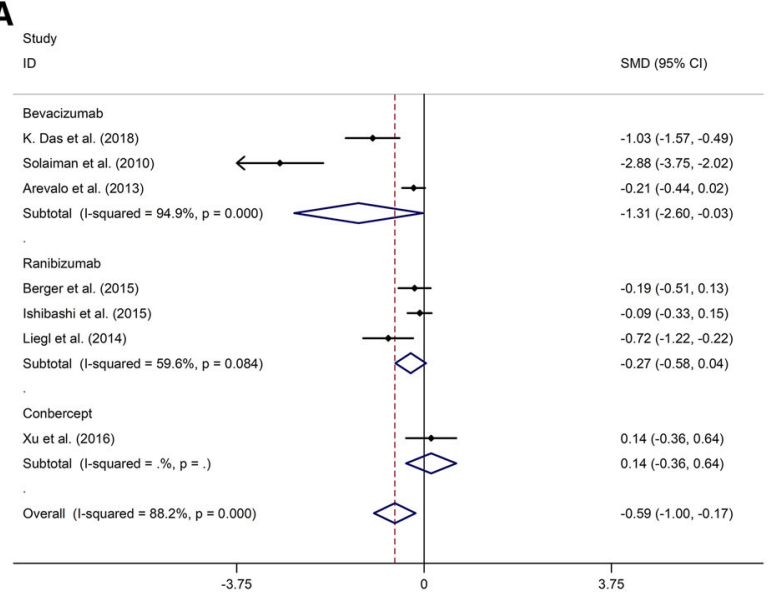

C

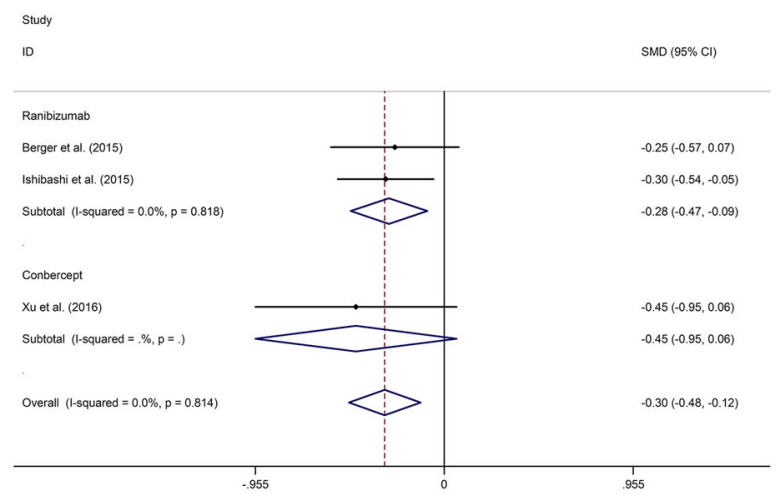

Fig. 3 Subgroup studies of CMT comparing patient groups (DME patients given combination therapy with an anti-VEGF agent and MPC vs. those receiving monotherapy with an anti-VEGF agent) receiving different kinds of anti-VEGF agents at 3 (a), 6 (b), 9 (c) and 12 they showed that post-treatment CMT was lower at 6, 9 and 12 months in DME patients given combination therapy with ranibizumab and MPC than in DME patients given monotherapy with ranibizumab. Moreover, there were no significant differences in posttreatment BCVA at 1, 3, 6, 9 and 12 months between DME patients receiving combination therapy with an anti-VEGF agent (bevacizumab or ranibizumab) and MPC and those receiving monotherapy with an anti-VEGF agent.

The results of our meta-analysis also showed that post-treatment CMT at 3 months was lower in DME patients given combination therapy with bevacizumab and MPC than in those

B
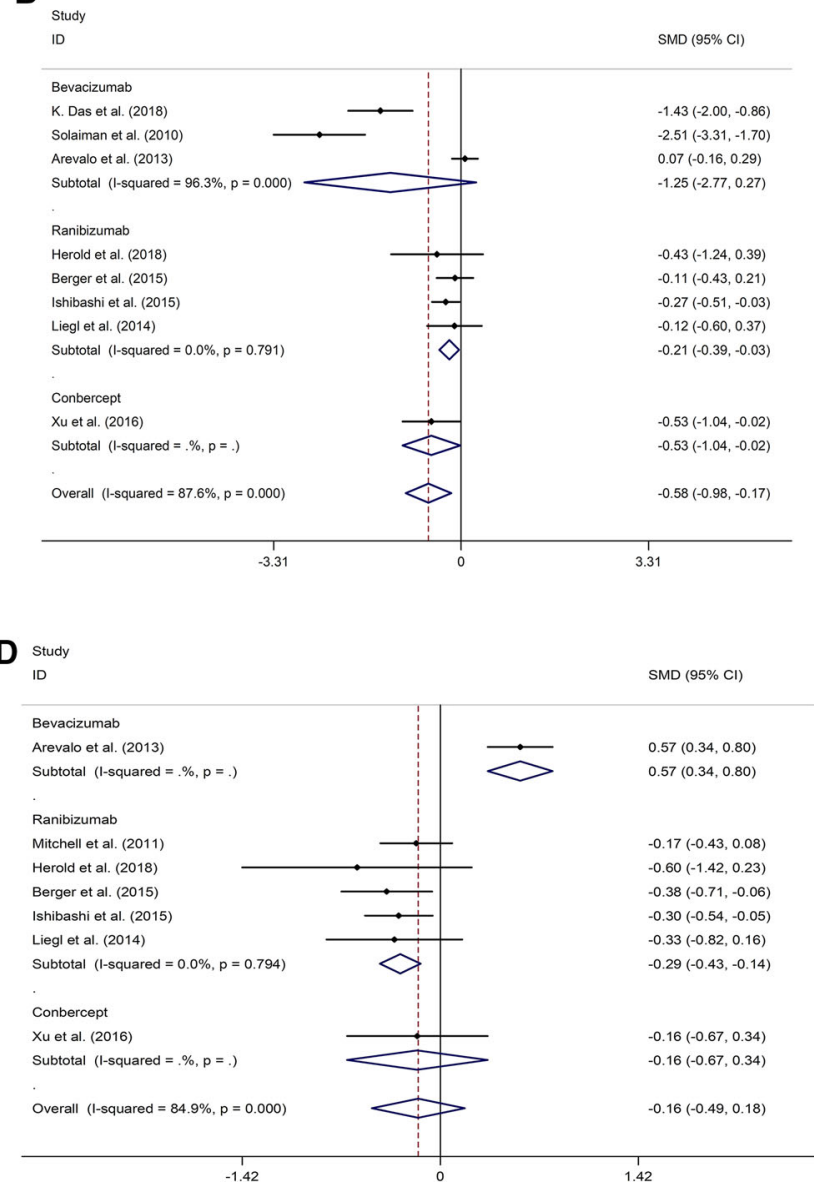

(d) months of follow-up. Diamonds denote WMD and 95\% CI. Negative values indicate lower CMT in DME patients given combination therapy with an anti-VEGF agent and MPC than in DME patients receiving monotherapy with an anti-VEGF agent 


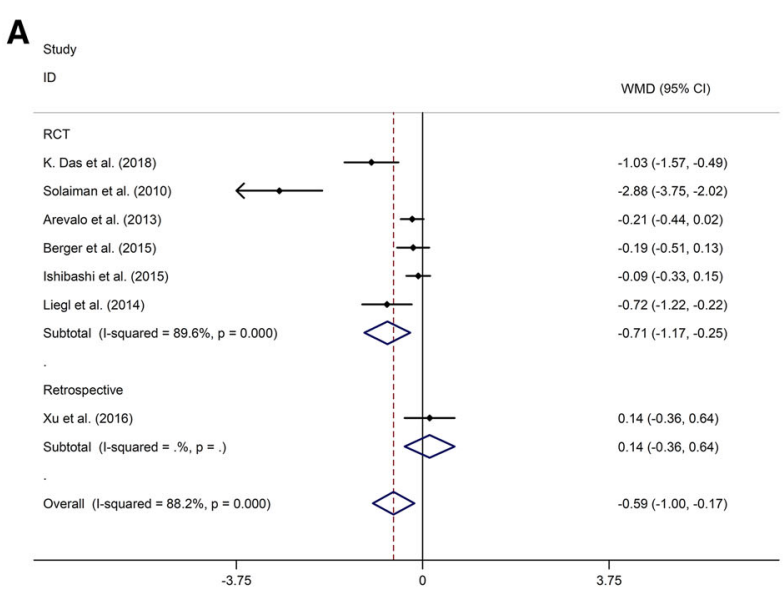

C

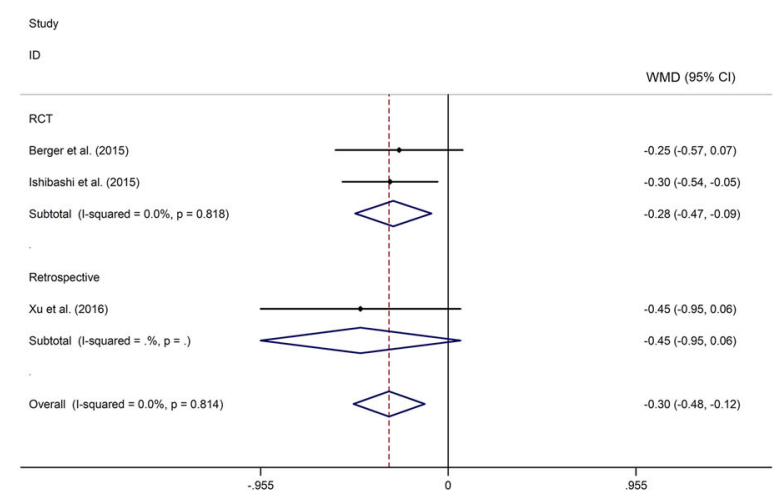

Fig. 4 Subgroup studies of CMT comparing patient groups (DME patients given combination therapy with an anti-VEGF agent and MPC vs. those receiving monotherapy with an anti-VEGF agent) in studies with different designs (randomized controlled trial $[R C T]$ vs. retrospective study) at $3(\mathbf{a}), 6(\mathbf{b}), 9(\mathbf{c})$ and $12(\mathbf{d})$ months

receiving monotherapy with bevacizumab. This result is inconsistent with those reported by the authors of a recent meta-analysis who found no significant differences in post-treatment CMT between two groups of DME patients at 1, 3 and 6 months [17]. One explanation for this difference may be that the present meta-analysis included the recently published article of Das et al. [13] that was not included in the earlier meta-analysis. Das et al. [13] showed that the DME patient group receiving combination therapy had significantly lower post-treatment CMT at 3 and 6 months than did the DME
B

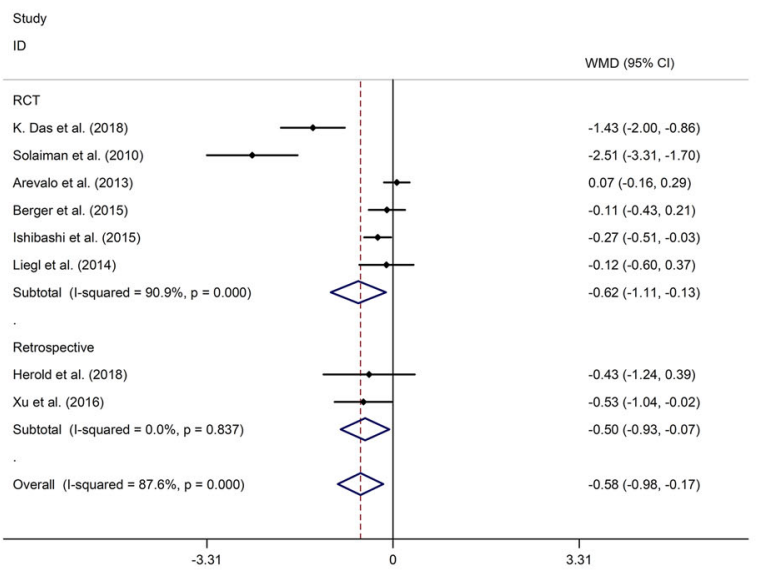

D

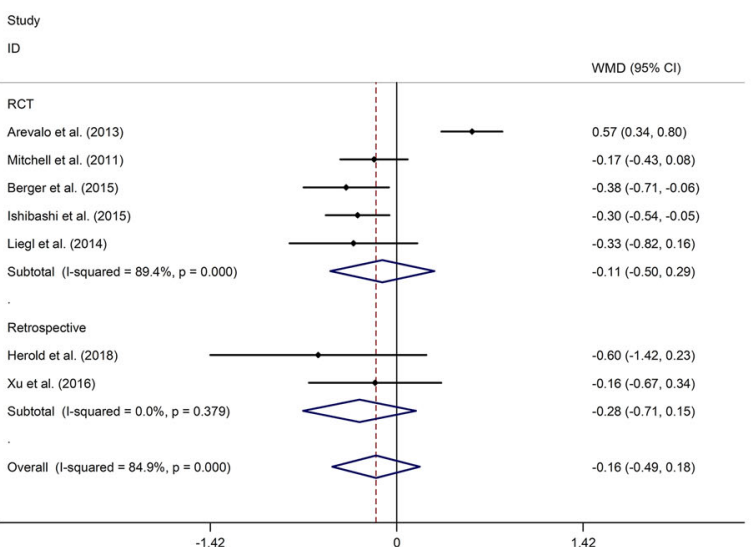

of follow-up. Diamonds denote WMD and 95\% CI. Negative values indicate lower CMT in DME patients given combination therapy with an anti-VEGF agent and MPC than in DME patients receiving monotherapy with an anti-VEGF agent

patient group receiving monotherapy with intravitreal bevacizumab. Solaiman et al. [9] indicated that combined therapy with

Fig. 5 Forest plot of best-corrected visual acuity (BCVA) comparing patient groups (DME patients given combination therapy with an anti-VEGF agent and MPC vs. those receiving monotherapy with an anti-VEGF agent) at 1,3 , $6,9,12,18,24,30,32$ and 36 months of follow-up. Diamonds denote WMD and 95\% CI. Negative values indicate lower BCVA in DME patients given anti-VEGF agents combined with MPC than in DME patients receiving monotherapy with an anti-VEGF agent 
Study

ID

WMD $(95 \% \mathrm{Cl})$

1 month

Solaiman et al. (2010)

Arevalo et al. (2013)

Lee et al. (2011)

Jovanovic et al. (2015)

Subtotal (I-squared $=65.9 \%, p=0.032)$

3 months

K. Das et al. (2018)

Solaiman et al. (2010)

Arevalo et al. (2013)

Lee et al. (2011)

Nguyen et al. (2009)

Berger et al. (2015)

Ishibashi et al. (2015)

Liegl et al. (2014)

Xu et al. (2016)

Subtotal (I-squared $=86.5 \%, p=0.000$ )

6 months

K. Das et al. (2018)

Solaiman et al. (2010)

Arevalo et al. (2013)

Lee et al. (2011)

Herold et al. (2018)

Berger et al. (2015)

Ishibashi et al. (2015)

Liegl et al. (2014)

Xu et al. (2016)

Subtotal (I-squared $=67.7 \%, p=0.002$ )

9 months

Berger et al. (2015)

Ishibashi et al. (2015)

Xu et al. (2016)

Subtotal (I-squared $=59.8 \%, p=0.083$ )

12 months

Arevalo et al. (2013)

Mitchell et al. (2011)

Herold et al. (2018)

Berger et al. (2015

Ishibashi et al. (2015)

Liegl et al. (2014)

Xu et al. (2016)

Subtotal (I-squared $=83.3 \%, p=0.000$ )

18 months

Herold et al. (2018)

Subtotal (I-squared $=. \%, p=$.)

24 months

Arevalo et al. (2013)

Herold et al. (2018)

Subtotal ( $($-squared $=38.2 \%, p=0.203$ )

30 months

Herold et al. (2018)

Subtotal $(\mathrm{l}$-squared $=. \%, p=$.)

36 months

Herold et al. (2018)

Subtotal (I-squared $=. \%, p=$.)

Overall (I-squared $=74.9 \%, p=0.000$ )

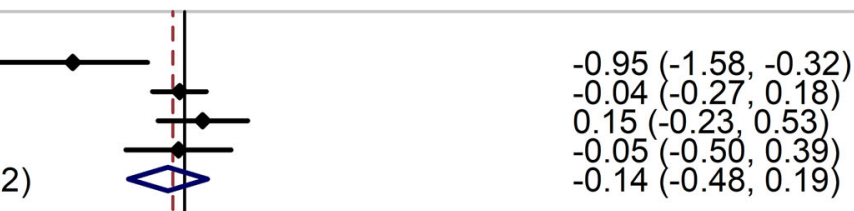

$0.43(-0.09,0.94)$

$-2.31(-3.09,-1.53)$

$-0.02(-0.25,0.20)$

$0.10(-0.28,0.48)$

$-0.12(-0.55,0.31)$

$-0.82(-1.15,-0.48)$

$0.14(-0.10,0.38)$

$-0.10(-0.58,0.38)$

$0.23(-0.28,0.73)$

$-0.21(-0.55,0.13)$

$0.42(-0.09,0.93)$

$-0.18(-0.78,0.42)$

$-0.07(-0.29,0.16)$

$0.21(-0.17,0.60)$

$0.47(-0.35,1.28)$

$-0.74(-1.07,-0.41)$

$-0.03(-0.27,0.21)$

$-0.20(-0.69,0.28)$

$0.23(-0.28,0.73)$

$-0.03(-0.27,0.20)$

$-0.34(-0.67,-0.02)$

$0.10(-0.14,0.34)$

$0.10(-0.40,0.61)$

$-0.05(-0.36,0.26)$

$0.00(-0.23,0.23)$

$0.69(0.42,0.95)$

$-0.40(-1.21,0.41)$

$-0.51(-0.84,-0.19)$

$0.02(-0.22,0.26)$

$-0.13(-0.61,0.36)$

$0.13(-0.37,0.63)$

$0.00(-0.31,0.32)$

$0.15(-0.65,0.95)$

$0.15(-0.65,0.95)$

$-0.11(-0.34,0.12)$

$-0.66(-1.49,0.16)$

$-0.24(-0.70,0.22)$

$-0.55(-1.37,0.27)$
$-0.55(-1.37,0.27)$

$-0.52(-1.34,0.29)$

$-0.52(-1.34,0.29)$

$-0.10(-0.22,0.02)$

\begin{tabular}{l|l|l|l} 
& & \\
\hline & & & \\
& -3.09 & 0 & 3.09
\end{tabular}


A

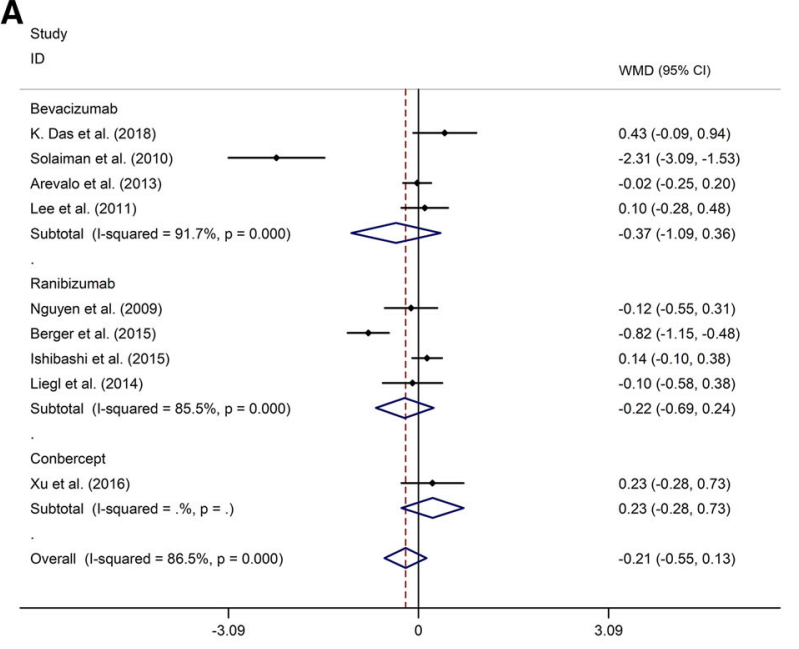

B

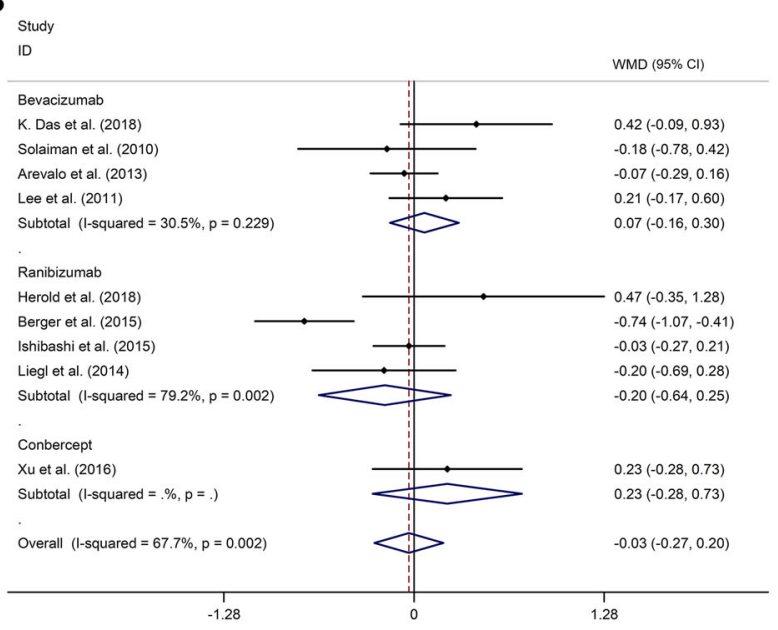

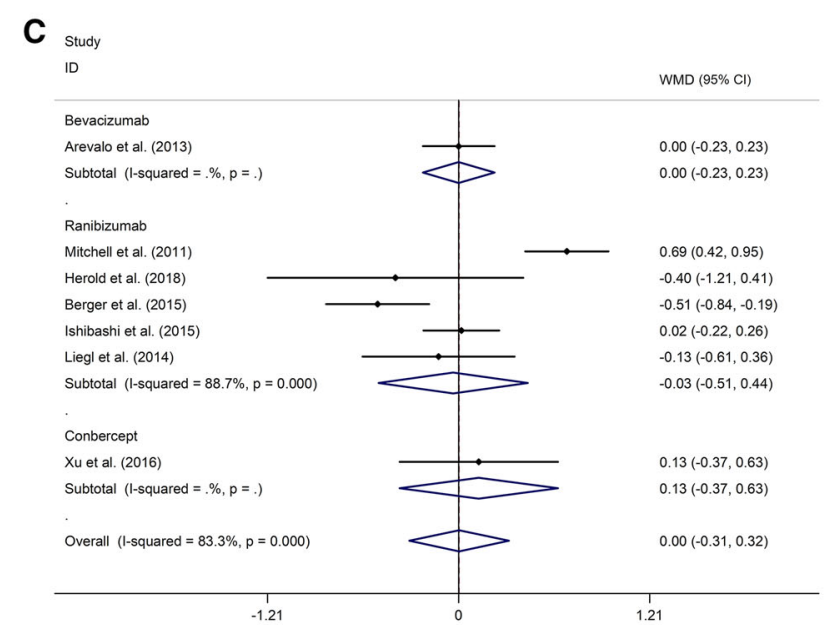

Fig. 6 Subgroup studies of BCVA comparing groups (DME patients given combination therapy with an antiVEGF agent and MPC vs. those receiving monotherapy with an anti-VEGF agent) receiving different kinds of antiVEGF agents at $3(\mathbf{a}), 6(\mathbf{b})$ and 12 (c) months. Diamonds denote WMD and 95\% CI. Negative values indicate lower BCVA in DME patients given anti-VEGF agents combined with MPC than in DME patients receiving monotherapy with an anti-VEGF agent

DME patients receiving monotherapy with ranibizumab and those receiving combination therapy with ranibizumab and MPC. Ishibashi et al. [19] reported that post-treatment CMT was lower in DME patients receiving combination therapy with ranibizumab and MPC at 6,9 and 12 months than in those receiving monotherapy with ranibizumab. Mitchell et al. [20] indicated no significant difference in posttreatment CMT between the two groups of DME patients at 12 months. Berger et al. [21] showed that combined therapy with ranibizumab and 
A

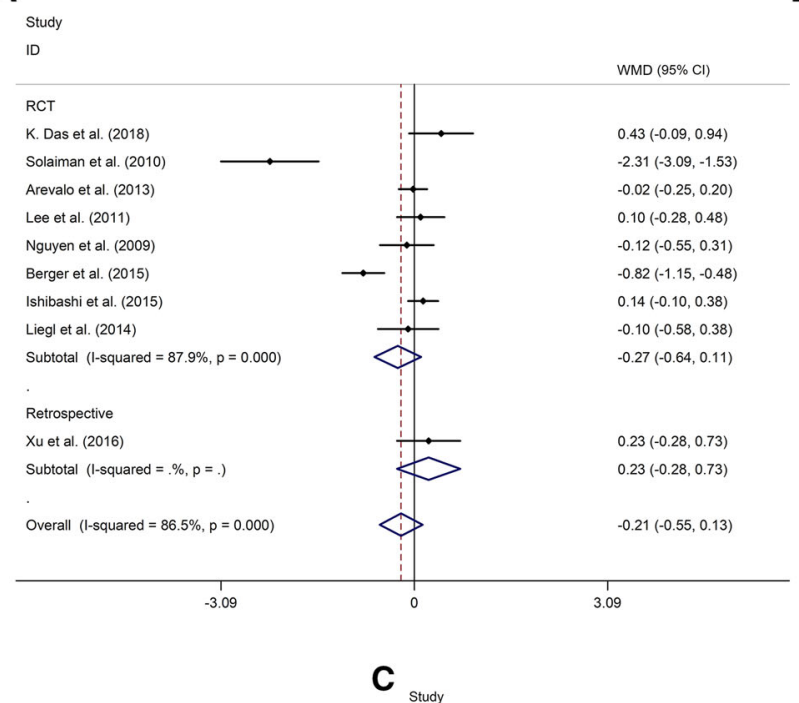

B

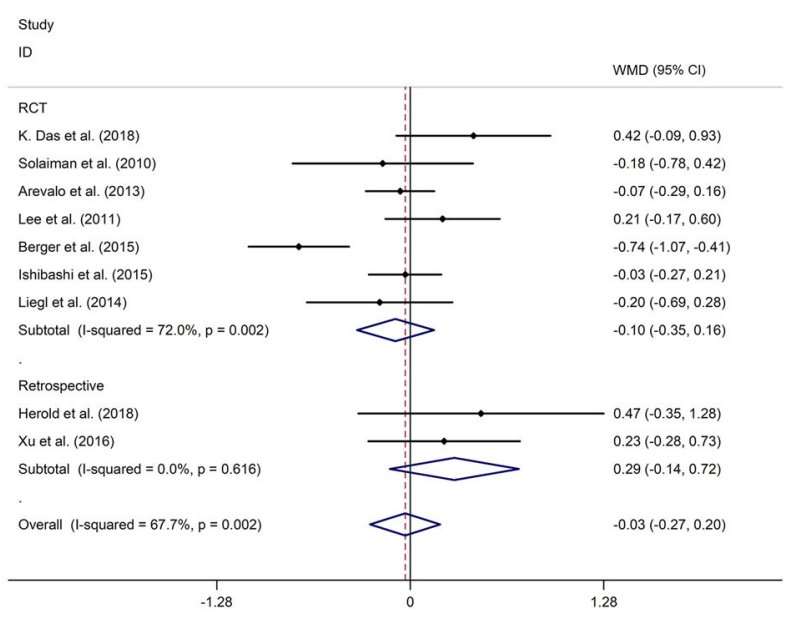

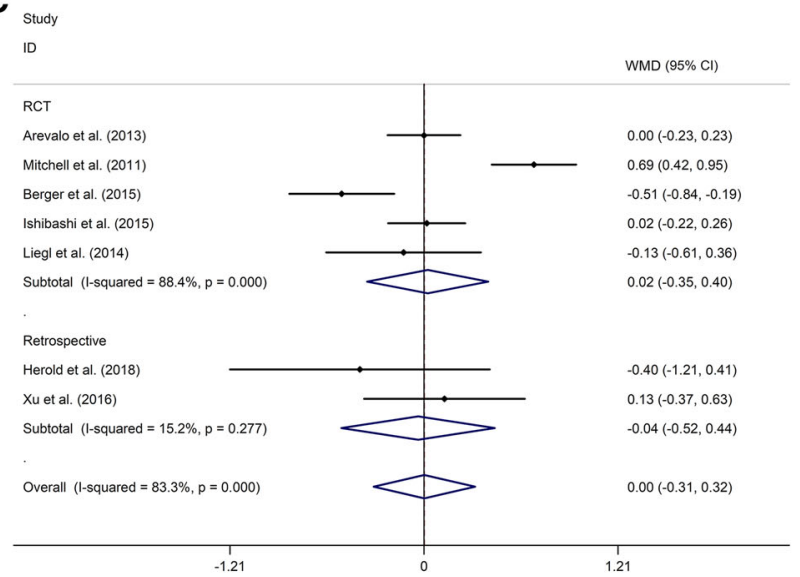

Fig. 7 Subgroup studies of BCVA comparing groups (DME patients given combination therapy with an antiVEGF agent and MPC vs. those receiving monotherapy with an anti-VEGF agent) in studies with different types of design at $3(\mathbf{a}), 6(\mathbf{b})$ and $12(\mathbf{c})$ months. Diamonds denote

MPC was superior to monotherapy with ranibizumab in terms of CMT reduction at 12 months, whereas there was no significant difference in post-treatment CMT between the two groups of DME patients at 3, 6 and 9 months. Our meta-analysis showed that CMT was lower at 6, 9 and 12 months of follow-up in DME patients given combination therapy with ranibizumab and MPC than in those receiving monotherapy with ranibizumab; however, the difference disappeared after 12 months of follow-up. CMT is an index that represents the anatomic change in DME levels. The better
WMD and 95\% CI. Negative values indicate lower BCVA in DME patients given anti-VEGF agents combined with MPC than in DME patients receiving monotherapy with an anti-VEGF agent

results in DME patients receiving combination therapy with an anti-VEGF agent and MPC indicate that MPC had a transiently synergistic effect in decreasing the level of DME when used in combination with an anti-VEGF agent. A relatively longer follow-up was essential to be able to evaluate the long-term existence of the synergistic effect.

In contrast, the meta-analysis showed no significant differences in BCVA at 1, 3, 6, 9 and 12 months of follow-up between DME patients receiving combination therapy with an antiVEGF agent and MPC and those receiving 
monotherapy with an anti-VEGF agent. This result corresponds to the findings of a recent meta-analysis [17] that showed no difference in BCVA at 1,3 and 6 months of follow-up between DME patients receiving combination therapy with bevacizumab and MPC and those receiving monotherapy with bevacizumab. This absence of any difference between the two groups did not parallel the corresponding changes in CMT in the eyes of the two patient groups, possible due to the many factors that can influence the visual outcome. Such factors include the severity and duration of macular edema, age of the patient, status of the macula before the onset of edema and presence of foveal hard exudates [22, 23]. These results support our finding of no synergistic effect of combined therapy with an anti-VEGF agent and MPC on BCVA.

There are some limitations to our study. First, the number of included studies was limited in order to explore the sources of heterogeneities. Second, some studies did not adjust for common confounding variables (the degree of glycemic control and level of serum lipids), which may have had an influence on the results of the meta-analysis.

\section{CONCLUSIONS}

In conclusion, the results of the present metaanalysis demonstrate a transiently synergistic effect of MPC on CMT when used in combination with an anti-VEGF agent, whereas no similar synergistic effect could be detected on BCVA. Also, a relatively longer follow-up was essential to be able to evaluate the existence of the synergistic effect.

\section{ACKNOWLEDGEMENTS}

Funding. No funding or sponsorship was received for this study or publication of this article. The article processing charges were funded by the authors.
Authorship. All named authors meet the International Committee of Medical Journal Editors (ICMJE) criteria for authorship for this article, take responsibility for the integrity of the work as a whole, and have given their approval for this version to be published.

Disclosures. On behalf of all authors, the corresponding author Bingtian Jiao states that there is no conflict of interest related to this study. The co-authors themselves, Lanjun Cui and Quanhong Han, also have nothing to disclose.

Compliance with Ethics Guidelines. This article is based on previously conducted studies and does not contain any studies with human participants or animals performed by any of the authors.

Data Availability. The datasets generated and/or analyzed during the current study are available from the corresponding author on reasonable request.

Open Access. This article is distributed under the terms of the Creative Commons Attribution-NonCommercial 4.0 International License (http://creativecommons.org/licenses/ by-nc/4.0/), which permits any noncommercial use, distribution, and reproduction in any medium, provided you give appropriate credit to the original author(s) and the source, provide a link to the Creative Commons license, and indicate if changes were made.

\section{REFERENCES}

1. Zhang X, Bao S, Lai D, Rapkins RW, Gillies MC. Intravitreal triamcinolone acetonide inhibits breakdown of the blood-retinal barrier through differential regulation of VEGF-A and its receptors in early diabetic rat retinas. Diabetes. 2008;57(4):1026-33.

2. Ding J, Wong TY. Current epidemiology of diabetic retinopathy and diabetic macular edema. Curr Diab Rep. 2012;12(4):346-54.

3. Keenan TDL, Johnston RL, Donachie PHJ, Sparrow JM, Stratton IM, Scanlon P. United Kingdom National Ophthalmology Database Study: diabetic 
retinopathy; report 1: prevalence of centre-involving diabetic macular oedema and other grades of maculopathy and retinopathy in hospital eye services. Eye. 2013;27(12):1397-404.

4. Patrao NV, Antao S, Egan C, et al. Real-world outcomes of ranibizumab treatment for diabetic macular edema in a United Kingdom National Health Service Setting. Am J Ophthalmol. 2016;172:51-7.

5. Bressler NM, Beaulieu WT, Glassman AR, et al. Persistent macular thickening following intravitreous aflibercept, bevacizumab, or ranibizumab for central-involved diabetic macular edema with vision impairment: a secondary analysis of a randomized clinical trial. JAMA Ophthalmol. 2018;136(3):257-69.

6. Kook D, Wolf A, Kreutzer T, et al. Long-term effect of intravitreal bevacizumab (avastin) in patients with chronic diffuse diabetic macular edema. Retina. 2008;28(8):1053-60.

7. Shimura M, Nakazawa T, Yasuda K, et al. Comparative therapy evaluation of intravitreal bevacizumab and triamcinolone acetonide on persistent diffuse diabetic macular edema. Am J Ophthalmol. 2008;145(5):854-61.

8. Soheilian M, Ramezani A, Bijanzadeh B, et al. Intravitreal bevacizumab (avastin) injection alone or combined with triamcinolone versus macular photocoagulation as primary treatment of diabetic macular edema. Retina. 2007;27(9):1187-95.

9. Solaiman KA, Diab MM, Abo-Elenin M. Intravitreal bevacizumab and/or macular photocoagulation as a primary treatment for diffuse diabetic macular edema. Retina. 2010;30(10):1638-45.

10. Krohne TU, Eter N, Holz FG, Meyer CH. Intraocular pharmacokinetics of bevacizumab after a single intravitreal injection in humans. Am J Ophthalmol. 2008;146(4):508-12.

11. Ahn SJ, Ahn J, Park S, et al. Intraocular pharmacokinetics of ranibizumab in vitrectomized versus nonvitrectomized eyes. Invest Ophthalmol Vis Sci. 2014;55(1):567-73.

12. Solaiman KA, Diab MM, Dabour SA. Repeated intravitreal bevacizumab injection with and without macular grid photocoagulation for treatment of diffuse diabetic macular edema. Retina. 2013;33(8):1623-9.

13. Das GK, Sahu PK, Biakthangi LVL, Jain D. Evaluation of intravitreal bevacizumab as monotherapy and in combination with macular grid laser photocoagulation in diffuse diabetic macular edema. Oman J Ophthalmol. 2018;11(3):248-53.
14. Arevalo JF, Lasave AF, Wu L, Diaz-Llopis M, Gallego-Pinazo R, Alezzandrini AA, et al. Intravitreal bevacizumab plus grid laser photocoagulation or intravitreal bevacizumab or grid laser photocoagulation for diffuse diabetic macular edema: results of the Pan-american Collaborative Retina Study Group at 24 months. Retina. 2013;33(2):403-13.

15. Lee SJ, Kim ET, Moon YS. Intravitreal bevacizumab alone versus combined with macular photocoagulation in diabetic macular edema. Korean J Ophthalmol KJO. 2011;25(5):299-304.

16. Moher D, Liberati A, Tetzlaff J, Altman DG, PRISMA Group TP. Preferred reporting items for systematic reviews and meta-analyses: the PRISMA statement. PLoS Med. 2009;6(7):e1000097.

17. Xiao K, Weng SJ, Liang SZ, Wang J, Qian C, Wan GM. Effect of intravitreal bevacizumab with or without macular photocoagulation for diabetic macular edema: a meta-analysis. Diabetes Ther. 2018;9(6):2369-81.

18. Javanović S, Canadanović V, Sabo A, Grgić Z, Mitrović M, Rakić D. Intravitreal bevacizumab injection alone or combined with macular photocoagulation compared to macular photocoagulation as primary treatment of diabetic macular edema. Vojnosanit Pregl. 2015;72(10):876-82.

19. Ishibashi $\mathrm{T}$, Li X, Koh A, et al. The REVEAL study: ranibizumab monotherapy or combined with laser versus laser monotherapy in Asian patients with diabetic macular edema. Ophthalmology. 2015;122(7):1402-15.

20. Mitchell P, Bandello F, Schmidt-Erfurth U, et al. The RESTORE study: ranibizumab monotherapy or combined with laser versus laser monotherapy for diabetic macular edema. Ophthalmology. 2011;118(4):615-25.

21. Berger A, Sheidow T, Cruess AF, Arbour JD, Courseau AS, de Takacsy F. Efficacy/safety of ranibizumab monotherapy or with laser versus laser monotherapy in DME. Can J Ophthalmol. 2015;50(3):209-16.

22. Eriksson U, Alm A, Bjarnhall G, Granstam E, Matsson AW. Macular edema and visual outcome following cataract surgery in patients with diabetic retinopathy and controls. Graefe's Arch Clin Exp Ophthalmol. 2011;249(3):349-59.

23. Otani T, Kishi S. Tomographic findings of foveal hard exudates in diabetic macular edema. Am J Ophthalmol. 2001;131(1):50-4.

24. Nguyen QD, Shah SM, Heier JS, et al. Primary end point (6 months) results of the ranibizumab for 
edema of the macula in diabetes (READ-2) study. Ophthalmology. 2009;116(11):2175-81 (e1).

25. Herold TR, Langer J, Vounotrypidis E, Kernt M, Liegl R, Priglinger SG. 3-year-data of combined navigated laser photocoagulation (Navilas) and intravitreal ranibizumab compared to ranibizumab monotherapy in DME patients. PLoS One. 2018;13(8):e0202483.
26. Liegl R, Langer J, Seidensticker F, et al. Comparative evaluation of combined navigated laser photocoagulation and intravitreal ranibizumab in the treatment of diabetic macular edema. PLoS One. 2014;9(12):e113981.

27. $\mathrm{Xu} \mathrm{Y,} \mathrm{Rong} \mathrm{A.} \mathrm{Intravitreal} \mathrm{conbercept} \mathrm{injection}$ with and without grid laser photocoagulation in the treatment of diffuse diabetic macular edema in real-life clinical practice. J Ophthalmol. 2016;2016:2143082. 